TNASA-TY-X-66206) THEORY OF THE 1973 ANOMALY IN THE THERMOSPH. OF THE PHASE HC $\$ 3.75$ THE THERMOSPHERE (NASA): 34 $\operatorname{CSCL}$. $04 \mathrm{~A}$ 
CONTENTS

Page

ABSTRACT. ............................. v

I. INTRODUCTION ............................. 1

II. OBSERVATIONS ............................. 3

a. Radar Temperature......................... 3

b. Satellite Drag Data ........................ 4

c. Satellite Spectrometer Data. ................... 5

III. THEORY .............................. 6

a. Semidiurnal and Terdiurnal Components ............. 8

b. Height Dependence in the Phases of the Energy Input Component . 9

c. Non Linear Coupling Between the Diurnal and Semidiurnal Modes .................................. 10

d. Energy Coupling From the Lower Atmosphere . . . . . . . . . 10

e. Latitude Dependence in the Diurnal Variations ........... 11

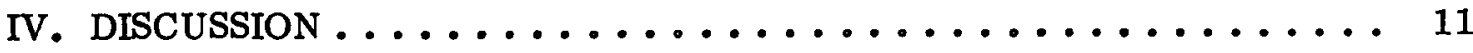

a. Diffusion and Energy Advection .................. 12

b. Energy Source Below $120 \mathrm{~km}$................... 15

c. Higher Harmonics ........................... 16

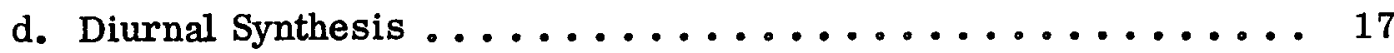

V. CONCLUSION............................... 20

VI. REFERENCES. .............................. 22

PRECEDINT; TA $\because$ RIAANR NUT MI!NG: 


\section{THEORY OF THE PHASE ANOMALY IN THE THERMOSPHERE}

$$
\text { by }
$$

H. G. Mayr, I. Harris and H. Volland*

Thermosphere and Exosphere Branch

February 1973

* University of Bonn, Germany

Goddard Space Flight Center

Greenbelt, Maryland 


\section{ABSTRACT}

The temperature-density phase anomaly is discussed on the basis of a quasi three dimensional model in which the thermosphere dynamics (including energy advection and diffusion associated with wind circulation) is considered in a self consistent form. Included in this analysis are the first three harmonics with non linear coupling between diurnal and semi diurnal tides. It is shown that the phase anomaly, with the exospheric temperature peak near $16^{00} \mathrm{LT}$ and the mass density peak between $14^{00}$ and $14^{45} \mathrm{LT}$, can be reproduced in a self consistent theory without invoking ad hoc assumptions or boundary conditions that normally mask the physical processes to be explored. It is concluded that the following factors and processes contribute to the phase anomaly: a) the importance of the semi- and particularly the ter-diurnal components which tend to shift the temperature peak toward the late afternoon thus accounting for the relatively good agreement with radar backscatter measurements, b) the effects from heat advection which can produce a phase shift in the temperature toward $12^{00} \mathrm{LT}$ in the lower thermosphere causing the density to peak earlier than the temperature higher up, where the temperature peaks later on in the afternoon, c) the effects from diffusion which redistributes $O$ (and $\mathrm{He}$ ) such that these constituents peak significantly earlier than $\mathrm{N}_{2}$ and $\mathrm{T}_{\mathrm{g}}$ at $200 \mathrm{~km}$ thereby contributing most effectively to the temperature density phase difference at 
exospheric heights and d) the energy coupling with the lower atmosphere which enhances the thermospheric wind velocities and with that amplifies the influence of heat advection and diffusion in producing the phase anomaly. 


\section{THEORY OF THE PHASE ANOMALY \\ IN THE THERMOSPHERE}

\section{INTRODUCTION}

The observed phase difference between the thermospheric radar temperature (Carru et al. (1967), Mahajan (1969), Waldteufel and McClure (1969), Nisbet (1970) and Salah and Evans (1973)) and the satellite drag density (Jacchia and Slowey (1965), Jacchia (1970)) is commonly regarded as anomalous. A number of suggestions have been made to account for this phenomenon (Rishbeth (1969), Chandra and Stubbe (1970), Mayr and Volland (1972, 1973) and Volland and Mayr (1973)), but a consensus on the origin of this effect has not been reached.

Rishbeth (1969) suggested that horizontal winds could cause an appreciable density depletion above $200 \mathrm{~km}$ thus shifting the density peak toward earlier local times. Chandra and Stubbe (1970) adopted a procedure to describe the phase discrepancy within a limited altitude range. They neglected all dynamic effects that are associated with the atmospheric circulation and solved the one dimensional heat conduction equation which-in agreement with Harris and Priester (1962) - produces a temperature peak near $17^{00}$ LT. However, deviating from Harris and Priester, who argued that the long charactersitic time for energy transport would not permit significant density and temperature variations in the lower thermosphere, Chandra and Stubbe adopted for their boundary conditions at $120 \mathrm{~km}$ an artificial factor of three variation in the density with the

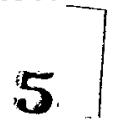


peak occuring at $12^{00} \mathrm{LT}$. Combining this early density maximum with the effect from the late temperature peak, Chandra and Stubbe then reproduced under the assumption of diffusive equilibrium - the density maximum at around $14^{00} \mathrm{LT}$ between 200 and $300 \mathrm{~km}$ altitude. This result essentially confirms Harris and Priester (1965) who varied the boundary conditions at $120 \mathrm{~km}$ for $\mathrm{O}, \mathrm{T}_{\mathrm{g}}$ and the $\mathrm{O} / \mathrm{O}_{2}$ ratio separately and concluded that with such a procedure the diurnal density variations can be fitted only within a narrow altitude range (around $600 \mathrm{~km}$, which at that time was considered representative for the satellite drag data) while producing poor agreement at other altitudes.

It was shown by Mayr and Volland (1972) that in the diurnal variations the thermospheric circulation can produce substantial deviations from diffusive equilibrium for atomic oxygen below $200 \mathrm{~km}$, thereby shifting its density peak away from the temperature and toward earlier local times. This effect is most pronounced within the lower thermosphere but it becomes gradually masked by the thermal expansion at greater heights such that the phase difference between temperature and mass density can decrease from about $3 \mathrm{hrs}$ at $160 \mathrm{~km}$ toward $1 \mathrm{hr}$ at $400 \mathrm{~km}$. However, He the major constituent at greater heights is even more affected by the diffusion process than $O$ thus reaching its diurnal maximum already during late morning hours (Mayx and Volland (1973)) with the consequence that it can further contribute to maintain a fiaite phase difference between temperature and mass density at exospheric heights. Although the OGO-6 composition measurements (Hedin et al. 1973) seem to confirm the phase se- 
quence of $\mathrm{He}, \mathrm{O}$ and $\mathrm{N}_{2}$ as predicted in our diffusion models a number of other processes can further contribute to the phase anomaly. One such process was discussed in Mayr and Volland (1973) where it was shown that the energy transport associated with the thermospheric circulation (particularly significant in the lower thermosphere where ion drag is relatively insignificant) can produce a height dependence in the temperature phase such that the temperature and density maxima are shifted toward earlier local times at lower altitudes as a result of which the density can peak earlier than the temperature in the upper thermosphere. Volland and Mayr (1973), finally, showed that tidal waves leaking from below into the thermosphere can further contribute to the phase discrepancy.

Considering these dynamic properties of the thermosphere phase differences between density and temperature are thus basically understandable. At this point the question remains whether the combined effects from the above discussed processes can quantitatively account for the actual magnitude of the observed phase discrepancy.

II. OBSERVATIONS

Radar backscatter measurements, satellite drag data and in situ neutral composition measurements on OGO-6 (Carignan and Pinkus, 1968) are the primary sources which provide information on the phase anomaly in the thermosphere. Associated with each of these data sets are certain characteristics which could affect the apparent magnitude of the density-temperature phase discrepancy. 


\section{a. Radar Temperature}

By consideration of the photoelectron energy transfer between electron, ion and neutral components the radar backscatter measurements of plasma temperatures are utilized to infer the gas temperature. This method is generally recognized as a powerful means of obtaining the gas temperature. Nevertheless some reservations should still be voiced considering that relatively small errors of $10^{\circ}-20^{\circ} \mathrm{K}$ could substantially influence the magnitude of the density-temperature phase difference (see Fig. 1 in Schwartz et al (1972)). The discrepancies between the exospheric radar and probe measurements of $T_{e}$ (Carlson and Sayers (1971), Hanson et al. (1970)) are not resolved and it has to be seen whether the corrections for the grid transparency of ion traps (Hanson et al. (1972)) can fully account for the disagreement between the observed radar and trap measurements of $T_{i}$ (McLure and Troy (1971), Hanson et al. (1971)). Furthermore, it is questionable whether our understanding of the ionospheric energetics is far enough developed to warrant a relatively accurate determination of the gas temperature particularly in view of the uncertainties in certain energy sources for the ion component associated with the dissipation of electric currents (Cole 1971)) and thermospheric winds (Hanson and Sanatani (1970), Stubbe and Chandra (1971)). Furthermore, radar observations are made only at a few locations and thus cannot provide a global picture of the temperature field. On the other hand the time resolution in the radar measurements is practically unlimited and superior to that of the satellite drag and in situ density measurements. 


\section{b. Satellite Drag Data}

The decays of satellite orbits is interpreted in terms of integral effects from the drag interaction of the thermospheric mass density above $250 \mathrm{~km}$. These measurements are thus to some extent dependent on a preconceived picture of the thermosphere structure as reflected in the empirical models . used for the satellite drag analysis. Jacchia's model is characterized by the concepts of diffusive equilibrium, invariant densities at $120 \mathrm{~km}$ and by height and latitude independent density phases all apparently corroborated by the satellite drag data. Reasonable as these assumptions are in a first approximation, they are not completely justified on theoretical grounds and the question thus remains whether a more complex thermosphere with deviations from the above concepts could not be equally or even more compatible with the satellite drag observations. The dilemma in the description of the elongated equatorial pressure bulge (Jacchia (1965), Jacchia and Slowey (1967)) - a phenomenon recently confirmed by Hedin and Mayr (1973)-reflects upon the uncertainties inherent in the interpretation of satellite drag data.

\section{c. Satellite Spectrometer Data}

In situ mass spectrometer measurements on OGO-6 (Carignan and Pinkus, 1968) provide information on the composition (including $\mathrm{N}_{2}, \mathrm{O}$ and $\mathrm{He}$ ) at altitudes around $450 \mathrm{~km}$. The temperatures inferred from the $\mathrm{N}_{2}$ observations on OGO 6 (Hedin et al。1972) still rely on assumptions regarding the height distribution of the temperature and the invariance of temperature and $\mathrm{N}_{2}$ density at $120 \mathrm{~km}$, a weakness these data have in common with the "satellite drag temperatures." 
The "OGO-6 temperatures" are more realistic, however, in that $\mathrm{N}_{2}$ is very close to diffusive equilibrium. The OGO-6 in situ measurements should also provide better spatial and temporal resolutions in the density and temperature variations than the satellite drag data, at least until understanding of the thermosphere structure will lead to substantial improvements in the empirical models that are used for the interpretation of satellite drag.

\section{THEORY}

The theoretical model used in our analysis has been essentially described in Mayr and Volland (1973). The equations of mass, energy and momentum conservation are considered in a self consistent form with the inclusion of diffusive interaction (momentum transfer) between species. Two versions of the model were used: one describing the distributions of $\mathrm{O}$ and $\mathrm{N}_{2}\left(+\mathrm{O}_{2}\right)$, the major constituents up to $600 \mathrm{~km}$ and another one describing $\mathrm{He}$ as it diffuses through a fictitious component consisting of all the other species $\mathrm{O}, \mathrm{N}_{2}$ and $\mathrm{O}_{2}$. In our paper we shall not discuss He since it contributes only to the phase anomaly above $500 \mathrm{~km}$ (the upper boundary of our model). Ion drag, Coriolis and viscous forces are considered as well as heat conduction and energy advection associated with the thermospheric circulation.

The horizontal wind field is expressed in terms of vector spherical harmonics

$$
V_{\theta}=\sum_{m=-3}^{+3}\left[S(r) \frac{\partial P_{m}^{m}}{\partial \theta}+\frac{i m}{\sin \theta} R(r) P_{m+1}^{m}\right] e^{j m \omega \tau}
$$




$$
V_{\phi}=\sum_{m=-3}^{+3}\left[-R(r) \frac{\partial P_{m}^{m}+1}{\partial \theta}+\frac{i m}{\sin \theta} S(r) P_{m}^{m}\right] e^{j m \omega \tau}
$$

where

$\mathrm{v}_{\theta}, \mathrm{v}_{\phi}=$ are the meridional and zonal velocity components.

$\omega=$ angular frequency with the period of one day.

$\tau=(\omega t+\phi)=$ local time, with $t$ universal time and $\phi$ longitude.

$\theta=$ colatitude.

$\mathrm{P}_{\ell}^{\mathrm{m}}=$ spherical harmonics.

$S(r), R(r)=$ solenoidal and rotational velocity components.

$r$ = radial dependence.

One can easily verify that the divergence of the horizontal velocity, which enters into the equations of energy and mass conservation, only depends on the solenoidal component S (r):

$$
\operatorname{div}_{\text {hor }} \overrightarrow{\mathrm{V}} \equiv \operatorname{div}_{\text {hor }} \overrightarrow{\mathrm{V}}(\mathrm{S}(\mathrm{r}))
$$

In our model we thus neglect the rotational component $R(r)$, and determine $S(r)$ and

$$
V_{\phi} \simeq \sum_{m=-3}^{+3} \frac{i m S(r)}{\sin \theta} P_{m}^{m} e^{j m \omega \tau}
$$

from the horizontal momentum equation in the $\phi$ direction. The velocity

$$
\mathrm{v}_{\theta} \simeq \sum_{\mathrm{m}=-3 .}^{+3} \mathrm{~S}(\mathrm{r}) \frac{\partial \mathrm{P}_{\mathrm{m}}^{\mathrm{m}}}{\partial \theta^{\cdot}} \mathrm{e}^{\mathrm{jm} \omega \tau}
$$


is thereby considered for the Coriolis force term. By neglecting the meridional momentum equation the model is two dimensional in the mathematical sense, but has quasi three dimensional character in that the meridional component (5) is included (through (3)) in the continuity equations.

The analysis of Mayr and Volland (1973) will be expanded in our paper to include a) semidiurnal and terdiurnal components, b) height dependence in the phases of the energy input components, c) non linear coupling between the diurnal and semidiurnal modes, d) energy input below $120 \mathrm{~km}$ and e) latitude dependence in the diurnal variations. In the interest of conciseness these features are only briefly discussed.

a. Semidiurnal and Terdiurnal Components

The absolute magnitude of the solar energy input into the thermosphere is considered to be uncertain and it is determined by matching the theoretical temperature amplitudes with observations. The ratio between the Fourier components of the solar heat input, however, is assumed to be known. Following Volland and Mayr (1972) the heat input $Q$ is described in terms of spherical harmonics

$$
Q=\sum_{m=-3}^{3} \mathrm{q}_{m}^{m} \mathrm{P}_{m}^{m} \mathrm{e}^{j \mathrm{~m} \omega \tau}
$$

The relative amplitudes of these components are adopted from a Fourier analysis of the equatorial heat input distribution computed for the Harris and Priester (1962) model: 


$$
\begin{aligned}
& \frac{\mathrm{q}_{1}^{1} \text { (diurnal) }}{\mathrm{q}_{2}^{2}(\text { s emidiurnal })} \sim \frac{1}{7} \\
& \frac{\mathrm{q}_{3}^{3}(\text { terdiurnal })}{\mathrm{q}_{2}^{2}(\text { s emidiurnal })} \sim \frac{1}{5}
\end{aligned}
$$

It was assumed that these ratios are altitude independent an assumption not strictly justified. However, in view of the complexity in the heat input mechanisms which involve the ionized component of the thermosphere we accept this simplification for the time being.

b. Height Dependence in the Phases of the Energy Input Components

The first harmonic in the diurnal variation of the solar heat input has two contributions, one due to the zenith angle effect in the absorbed energy which peaks close to $12^{00}$ local time and a second one which results from the diurnal variation in the density which is particularly significant at higher altitudes . where the density amplitude becomes large. Since the phase in the diurnal density component occurs between $14^{00}$ and $15^{00} \mathrm{LT}$ the superposition of both contributions produces a phase shift in the maximum of the diurnal heat input component from $12^{00} \mathrm{LT}$ at lower altitudes toward about $13^{30}$. at higher altitudes. The non linear coupling between both components contributes to the second (semidiurnal) harmonic in the heat input such that its phase varies between $12^{00} \mathrm{LT}$ at lower altitudes and about $14^{00} \mathrm{LT}$ at higher altitudes. For moderate zenith angles (or for day time conditions) absorption effects are relatively insignificant and thus the heat input maximum is rather broad and 
elongated around noon. This particular characteristic is primarily responsible for the relatively strong third harmonic in the heat input (by comparing (6) and (7) note that the spherical harmonics associated with the coefficients $q_{1}^{1}, q_{2}^{2}, q_{3}^{3}$ have the forms $\sin \theta, 3 \sin ^{2} \theta, 15 \sin ^{3} \theta$ respectively) which has a nearly height independent phase close to $16^{00} \mathrm{LT}$. It will be shown later on that this terdiurnal component in the solar heat input significantly contributes to the apparent phase discrepancy between radar temperature and satellite drag density.

c. Non Linear Coupling Between the Diurnal and Semidiurnal Modes

It was shown by Volland and Mayr (1973) that the non linear coupling between the diurnal components in the ion drag and in the horizontal wind field cannot be neglected for the semidiurnal mode. In our analysis we assumed a 50\% relative amplitude for the diurnal electrondensity variation with the diurnal maximum occuring at $15^{00} \mathrm{LT}$. Also considered was the non linear coupling between a) the diurnal components of the neutral constituents and the wind field for the ion drag term in the horizontal momentum equation and b) the diurnal components of the partial pressures and the wind velocity which describe the mechanical work in the energy equation.

d. Energy Coupling From the Lower Atmosphere

Volland and Mayr $(1970,1973)$ discussed the diurnal tides in terms of characteristic waves and showed that energy coupling from the lower atmosphere can be of importance for the thermosphere dynamics. Rocket measurements of the thermospheric temperature distribution, analyzed by Newton and Mayr (1973), 
seem to confirm this. Deviating from an earlier paper (Mayr and Volland, 1973) we shall therefore extend the heat input from $120 \mathrm{~km}$ down to $90 \mathrm{~km}$ (the lower boundary of the model) as illustrated in Figure 1.

e. Latitude Dependence in the Diurnal Variations

With increasing latitude the amplitudes of the heat input decrease in our model like $\sin \theta, \sin ^{2} \theta$ and $\sin ^{3} \theta$ for the diurnal, semidiurnal and terdiurnal components respectively. The divergences of the horizontal wind field, which describe to a great extent the mass and energy loss rates in the continuity equations, would follow the same dependence if the electron density (ion drag) were globally uniform. With the electron density decreasing toward higher latitudes, however, the latitude dependence in the wind divergence is damped or even reversed thus affecting the global distribution of the density and temperature phases. For our model calculations we have chosen the electron density profiles shown in Figure 1 for $0^{\circ}$ and $45^{\circ}$ latitudes.

\section{DISCUSSION}

It will be shown later on that the semi-and ter-diurnal components contribute to only about $30 \%$ of the total temperature and density variations at the equator. Therefore we shall first concentrate on the diurnal component in discussing the various mechanisms that contribute to the phase anomaly.

Figures 2 through 4 show the height distributions for amplitudes and phases of $\mathrm{T}_{\mathrm{g}}, \mathrm{O}, \mathrm{N}_{2}$ and zonal wind velocity $\mathrm{V}$ computed under a number of assumptions for the equatorial region. In all three figures the dashed lines describe a 
common "standard condition" which serves as reference. It is characterized as follows: Momentum transfer between $\mathrm{O}$ and $\mathrm{N}_{2}$ (diffusion) is considered. The heat source is of the type shown in Figure 1 but without heat input below $120 \mathrm{~km}$. For ion drag a normal electron density distribution, shown as solid line in Figure 1, is adopted. With these inputs the equations of mass, energy and momentum conservation are solved to derive the various parameters.

The effects of diffusion, energy advection and energy coupling from the lower atmosphere below $120 \mathrm{~km}$ are illustrated in comparison with this normal distribution. Each of these three effects will be shown to contribute substantially to the phase anomaly.

a. Diffusion and Energy Advection

The diffusive equilibrium solution shown in solid lines in Figure 2 is derived by artificially setting the drag coefficient for the momentum transfer between $\mathrm{O}$ and $\mathrm{N}_{2}$ zero. The marked differences between diffusion ("standard condition') and diffusive equilibrium solutions, illustrated in Figure 2, were already discussed in some detail by Mayr and Volland $(1972,1973)$. It is the thermospheric circulation which is primarily responsible for this effect causing a diffusive redistribution of $\mathrm{O}$ (and $\mathrm{He}$ ) such that its density is substantially enhanced during morning hours below $250 \mathrm{~km}$. As a consequence, a significant phase difference of about one hour between $O$ and $T_{g}$ developes at exospheric heights (dashed 
lines) in contrast to the diffusive equilibrium solution (solid lines) for which the phase difference is substantially smaller.

Nevertheless, even in the diffusive equilibrium solution the $\mathrm{O}$ and $\mathrm{N}_{2}$ density maxima precede the temperature maximum in the upper thermosphere. This feature can be attributed to the early temperature maximum (around $12^{00} \mathrm{LT}$ ) below $160 \mathrm{~km}$ which produces there a corresponding phase shift in the thermospheric density toward earlier local times. At greater heights this effect is increasingly masked by the density dependence on the later temperature phase.

This particular height dependence in the temperature phase is caused by the advective energy redistribution associated with the thermospheric wind circulation in the lower thermosphere as illustrated in Figure 3. Here we have artificially increased the electron density in the $\mathrm{E}$ and lower $\mathrm{F}$ region (dashed line in Figure 1). As a result of this the horizontal wind is significantly damped which is apparent from the comparison between the velocity distributions in dashed and solid lines. This decrease in the wind velocity has a number of consequences: The energy advection - effectively an energy loss - associated with the wind circulation is also damped. With the energy loss rate decreasing the temperature amplitude thus grows (note the 30\% increase in the lower thermosphere) and the phase of the temperature maximum is shifted toward later local times. While for the "standard condition" (dashed line) the temperature maximum is set back from $14^{00} \mathrm{LT}$ at exospheric heights toward $12^{40} \mathrm{LT}$ at $120 \mathrm{~km}-$ 
thus contributing to the phase anomaly as pointed out earlier - with increased ion drag the phase distribution shows just the opposite trend in that it increases from $15^{20} \mathrm{LT}$ in the exosphe re toward $16^{40}$ at $120 \mathrm{~km}$. In diffusive equilibrium this kind of phase distribution would cause the density maxima to occur after the temperature maximum in reversed analogy to the case described in Figure 2. This is indeed apparent in Figure 3 for $\mathrm{N}_{2}$ a constituent close to diffusive equilibrium. The situation is different, however, for atomic oxygen when diffusion is considered. Even with the temperature maximum advanced to later local times at lower altitudes, the diffusive redistribution is still effective enough to maintain an oxygen peak that precedes the temperature maximum in the upper thermosphere. The phase in the mass density would thus change over from following the temperature at lower heights where $\mathrm{N}_{2}$ dominates toward preceding the temperature at higher altitudes where $O$ dominates. In this particular case, though, a phase difference of only a fraction of an hour can be attained at exospheric heights.

Contributing to this small phase discrepancy is still another factor that is related to the increased ion drag. With the resulting decrease in the horizontal wind velocity the diffusion velocity is also substantially (almost proportionally) decreased. The diffusive redistribution is thus much less significant in the solution with increased ion drag and this is also to a great extent responsible for the relatively small temperature-density phase difference in the exosphere as shown in solid lines in Figure 3. 


\section{b. Energy Source Below $120 \mathrm{~km}$}

The effects of energy coupling from the lower atmosphere are illustrated in Figure 4. The most obvious ones are that the horizontal velocity is significantly enhanced throughout the thermosphere as a result of the increase in the temperature amplitude at lower heights which in turn enhances the density variations. Associated with the velocity increase is also on enhancement in the advective energy exchange rate and thus the temperature amplitude is slightly damped above $160 \mathrm{~km}$. The wind circulation excited by the energy input below $120 \mathrm{~km}$ penetrates into the upper thermosphere and acts there essentially as an energy sink for the diurnal temperature variation. The relative increase in the advective energy redistribution (loss) is also apparent from the phase shift in the temperature toward earlier local times. This phase shift is particularly effective below $160 \mathrm{~km}$ where the temperature amplitude is significantly increased and with that it is responsible for the phase difference between $\mathrm{N}_{2}$ and $\mathrm{T}_{\mathrm{g}}$ at exospheric heights.

Due to the larger wind velocities induced by the energy input below $120 \mathrm{~km}$ the diffusive redistribution for $O$ becomes also more important. Thus the relative oxygen concentration is substantially enhanced above $160 \mathrm{~km}$ and its phase is shifted further away from the temperature thereby maintaining a phase difference of about an hour at altitudes as high as $440 \mathrm{~km}$.

Summarizing these case studies it can be said that transport associated with the thermospheric circulation is the common mechanism that induces in 
various ways the temperature-density phase anomaly. Energy advection which affects the phase distribution of the temperature is a factor in determining the phase difference between $\mathrm{T}_{\mathrm{g}}$ and $\mathrm{N}_{2}$ the major constituent below $250 \mathrm{~km}$. Oxygen is also affected by this process but it is diffusion which is primarily responsible for $\mathrm{O}-\mathrm{T}_{\mathrm{g}}$ phase difference and its maintenance at exospheric heights. Both energy advection and diffusion are significantly influenced by the distribution of the electron density (ion drag) and it appears that energy coupling from the lower atmosphere tends to amplify these processes.

c. Higher Harmonics

Amplitudes and phases of $\mathrm{O}, \mathrm{N}_{2}$ and $\mathrm{T}_{\mathrm{g}}$ are shown in Figure 5 for the semidiurnal and ter-diurnal components at the equator. In these solutions the energy input below $120 \mathrm{~km}$ is considered hence they are analogous to the diurnal component shown with solid lines in Figure 4.

Since the semidiurnal tide is partially excited by non linear coupling from the diurnal mode a comparison with diurnal and ter-diurnal tides (which are solely excited by solar heat input) is difficult. Nevertheless, it is characteristic for the higher harmonics that dynamic effects become increasingly more important. The reason for this is that the divergence of the horizontal wind velocity (or with other words the number of horizontal circulation cells) which enters into the continuity equations substantially increase (between a factor of two and 
three) from one to the next higher harmonics thus increasing the effectiveness of the horizontal mass and energy transport. Figure 5 reflects upon that.

The phase difference between $O$ and $T_{g}$, again primarily the result of wind induced diffusion, is about $45 \mathrm{~min}$ in the ter-diurnal component at $440 \mathrm{~km}$. Although this is only about the same time difference that developes in the diurnal component it is a three times larger fraction of the ter-diurnal time period which is a more appropriate quantity to compare with in discussing dynamic characteristics. By comparison similarly enhanced is the phase difference in $\mathrm{N}_{2}$ except that this species is more affected by the phase distribution in the temperature which in turn is greatly determined by the wind circulation.

The late peaks in the ter diurnal component of $\mathrm{O}, \mathrm{N}_{2}$ and $\mathrm{T}_{\mathrm{g}}$ are due to the $16^{00}$ LT phase in the solar heat input (see Chapter III).

Included in the semidiurnal tide is the non linear coupling between the diurnal variations of ion drag and wind velocity and its effect is essentially to decrease the semidiurnal wind velocity. Accordingly, the dynamic effects are somewhat less pronounced. Still the phase difference between oxygen and $\mathrm{T}_{\mathrm{g}}$ is about $30 \mathrm{~min}$ at $440 \mathrm{~km}$ which is comparable to that of the diurnal component considering the shorter time period.

d. Diurnal Synthesis

The temperature-density phase anomaly is, at least historically, tied to the time difference between the diurnal maxima in temperature and mass density 
(the quantity determined from satellite drag). In Figure 6 diurnal variations of temperature and mass density are synthesized from the diurnal, semidiurnal and ter diurnal components shown in Figure 4 (solid lines) and Figure 5. For comparison are also shown the diurnal variations computed for $45^{\circ}$ latitude. There, a factor of two lower electron density distribution was adopted (see Figure 1) to consider the latitudinal decrease in the concentration of the ionosphere plasma. We have chosen only the $450 \mathrm{~km}$ distribution for the temperature since it does not appreciably change down to $250 \mathrm{~km}$. For the mass density the relative variations are shown for both 450 and $250 \mathrm{~km}$. X marks indicate the location of the maxima. The dotted lines in the temperature distributions show the first harmonics, the dashed lines show the first and second harmonics and the solid lines show the sum of all three harmonics.

Considering that satellite drag data are derived from altitudes above $250 \mathrm{~km}$ it appears that our theory can reproduce the phase anomaly in the thermosphere when we look at the peaks in the diurnal variations of temperature and density. The temperature maxima occur at the equator near $16^{00} \mathrm{LT}$ and at $45^{\circ}$ latitude near $15^{30} \mathrm{LT}$, while the density maxima occur in both latitudes between $14^{00} \mathrm{LT}$ at $250 \mathrm{~km}$ and $14^{45} \mathrm{LT}$ at $450 \mathrm{~km}$. At higher altitudes the phase difference between $\mathrm{O}$ and $\mathrm{T}_{\mathrm{g}}$ will further continue to decrease. But above $500 \mathrm{~km}$ He can no longer be neglected especially since its diurnal maximum occurs substantially earlier than that in atomic oxygen (Hedin et al. 1973, Mayr and Volland, 1973) thus the addition of He will contribute to maintain the phase difference above $450 \mathrm{~km}$. 
The question remains whether a height dependence in the density phase of the kind suggested in Figure 6 is real. It has not been detected in the satellite drag measurements but their temporal and spatial resolution is limited and the empirical models used in describing these data are perhaps not sufficiently sophisticated considering the complexities associated with the thermosphere dynamics.

Our model calculations show essentially identical phases for the density variations at the equator and at $45^{\circ}$ latitude. Although thiṣ feature is in substantial agreement with the satellite drag data, which have not revealed any latitude dependence, theoretically there is no reason that would enforce this invariance. In our model it was brought about accidentally by the choice of electron density distributions at both latitudes. A higher or lower ion drag at $45^{\circ}$ latitude would have caused the density and temperature phases to be shifted toward later or earlier local times respectively. However, with increasing divergence of the horizontal wind field toward the equator the dynamic effects should become more prominent thus widening there the temperature-density phase difference particularly in the higher harmonics, an effect which is apparent in our results and which tends to stabilize the latitudinal variations in the density phase.

From the Fourier synthesis of the temperature variation it can be seen that in our theory the ter-diurnal component is to a great extent responsible for the late temperature peaks thus accounting for the relatively good agreement with the radar backscatter observations of the gas temperature. However, the magnitudes of the ter-diurnal (and semi diurnal) energy components are 
relatively small and are therefore most likely affected by non linear processes involving solar radiation and thermospheric and plasma densities. These complexities have not yet been considered in a self consistent form in our model and therefore a detailed comparison with radar backscatter temperatures and satellite drag densities is not very meaningful。

\section{CONCLUSION}

The temperature-density phase anomaly was discussed on the basis of a quasi three dimensional model in which the thermosphere dynamics (including energy advection and diffusion) associated with wind circulation was considered in a self consistent form. Included in the analysis were the first three harmonics which invloved the non linear coupling between diurnal and semidiurnal tides.

It was shown that the phase anomaly with the exospheric temperature peaks near $16^{00} \mathrm{LT}$ and the mass density peaks between $14^{00}$ and $14^{45} \mathrm{LT}$ can be reproduced in a self consistent theory without invoking ad hoc assumptions and boundary conditions that would mask the physical processes to be explored (our boundary conditions were homogeneous that is zero velocity, density-and temperature variations at the lower boundary $(90 \mathrm{~km})$ and zero temperature and velocity gradients at the upper boundary $(500 \mathrm{~km}))$. We conclude that a number of factors and processes contribute to the phase anomaly:

a) the importance of the semi- and in particular the ter-diurnal components tend to shift the temperature peak toward later local times thus accounting for the relatively good agreement with the radar backscatter measurements 
b) the effects from heat advection are particularly important in the lower thermosphere where this energy redistribution (loss) can shift the temperature phase back toward $12^{00}$ LT thus causing the density to peak before the temperature higher up where the temperature phase occurs in the afternoon.

c) most important perhaps are the effects from diffusion induced by the thermospheric circulation which redistribute $\mathrm{O}$ (and $\mathrm{He}$ ) such that these constituents peak significantly earlier than $\mathrm{N}_{2}$ and $\mathrm{T}_{\mathrm{g}}$ at $200 \mathrm{~km}$ thus contributing substantially to the temperature-density phase difference at exopsheric heights。

d) the energy input below $120 \mathrm{~km}$ can substantially enhance the wind field especially in the lower thermosphere, thereby amplifying the effects of heat advection and diffusion in producing the phase anomaly (see $b$ and $c$ ).

The theory presented here is relatively crude in the treatment of the thermosphere dynamics and one of its properties the "phase anomaly." The model is quasi three dimensional, and no attempts were made to explore or optimize under various conditions the effects that were shown to contribute to this phenomenon. With the uncertainties in the energy and ion drag distributions our analysis cannot be unique. It can only be said that density-temperature phase differences of the kind observed do not appear to constitute a problem considering certain properties of the thermosphere dynamics. In situ temperature and composition measurements are needed from the lower thermosphere to determine the relative importance of the various processes that were suggested here as causes for the phase anomaly. 


\section{REFERENCES}

1. Carignan, G.R. and W. H. Pinkus, OGO-F04 experiment description, University of Michigan Technical Note 08041-3-T, 1968.

2. Carlson, H. C. and J. Sayers, Discrepancy in electron temperatures deduced from Langmuir probe and from incoherent scatter radar, J. Geophys. Res., $75,4883,1970$.

3. Carru, H., M. Petit and P. Waldteufel, On the diurnal variation of the thermopause temperature, Planet. Space Sci., 15, 944, 1967.

4. Chandra, S. and P. Stubbe, The diurnal phase anomaly in the upper thermospheric density and temperature, Planet. Space Sci., 18, 1021, 1970.

5. Cole, K. D., Electrodynamic heating and movement of the thermosphere, Planet. Space Sci., 19, 59, 1971.

6. Hanson, W. B., L. H. Brace, P. L. Dyson, and J. P. McClure, Conflicting electron temperature measurements in the upper F-region, J.Geophys. Res., $74,400,1969$.

7. Hanson, W. B. and S. Sanatani, Meteoric ions above the $F_{2}$ peak, J. Geophys. Res., 75, 5503, 1970 。

8. Hanson, W. B., D. R. Frame, and J. E. Midgley, Errors in retarding potential analyzers by non-uniformity in the grid-plane potential, J. Geophys. Res., $77,1914,1972$ 。

9. Harris, I. and W. Priester, Time dependent structure of the upper atmosphere, J. Atmos. Terr. Phys., 29, 1429, 1967. 
10. Harris, I. and W. Priester, Of the diurnal variation of the upper atmosphere, J. Atmosp. Sci., 22, 3, 1965 .

11. Hedin, A. E. and H. G. Mayr, Magnetic control of the near equatorial neutral thermosphere, in press for J. Geophys. Res. 1973.

12. Hedin, A. E., H. G. Mayr, C. A. Reber, G. R. Carignan , and N. W. Spencer, A global empirical model of thermospheric composition based on OGO-6 mass spectrometer measurements, presented at the XV meeting of COSPAR, Madrid, Spain, 1972, in press for Space Res. XIII, 1973.

13. Jacchia, L. G. and J. Slowey, The shape and location of the diurnal bulge in the upper atmosphere, Space Res. VII, 1077, 1967.

14. Jacchia, L. G., Revised static models of the thermosphere and exosphere with empirical temperature profiles, Smithsonian Astrophysical Observatory, Special Report 332, 1971.

15. Mahajan, K. K., Diurnal variation of the ion temperature, J. Atmos. Terr. Phys., 31, 93, 1969 .

16. Mayr, H. G. and H. Volland, Diffusion model for the phase delay between thermospheric density and temperature, J. Geophys. Res., 77, 2359, 1972 .

17. Mayr, $H_{\circ}$ G. and $H_{0}$ Volland, Two component model for the diurnal variations in the thermospheric composition, J. Atm. Terr. Phys., 35,669 1973.

18. McClure, J. P. and B. E. Troy, $\mathrm{Jr}_{\bullet}$, Equatorial ion temperature: a comparison of conflicting incoherent scatter and OGO-4 retarding potential analyzer values, J. Geophys. Res., 76, 4531, 1971.

\section{2ary}


19. Newton, G. P. and H. G. Mayr, Diurnal and semidiurnal variations in the temperature and density from thermosphere probe measurements, submitted to J. Geophys. Res., 1973.

20. Nisbet, J. S., Neutral Atmospheric temperatures from incoherent scatter observations, J. Atmosph. Sci。, 24, 586, 1967.

21. Rishbeth, H., On the phase of the diurnal bulge in the thermosphere, Ann. Geophys., 25, 495, 1969.

22. Salah, J. E. and J. V. Evans, Measurements of thermospheric temperatures by incoherent scatter radar, presented at COSPAR, Madrid, 1972, in press for Space Res., XIII, 1973.

23. Schwartz, W. E., J. L. Kohrbaugh and J. S. Nisbet, A thermospheric model from satellite orbital decay densities and incoherent scatter temperatures, J. Atmos. Terr. Phys., 34, 1817, 1972.

24. Stubbe, P. and S. Chandra, Ionospheric warming by neutral winds, Planet. Space Sci., 19, 731, 1971.

25. Volland, H. and H. G. Mayr, A theory of the diurnal variations in the thermosphere, Ann. Geophys., 26, 907, 1970.

26. Volland, H. and H. G. Mayr, A numerical study of three dimensional diurnal variations in the thermosphere, in press for Ann. Geophys., 1973.

27. Waldteufel, P. and J. P. McClure, Preliminary comparisons of middle and low latitude Thomson scatter data, Ann. geophys., 25, 785, 1969. 


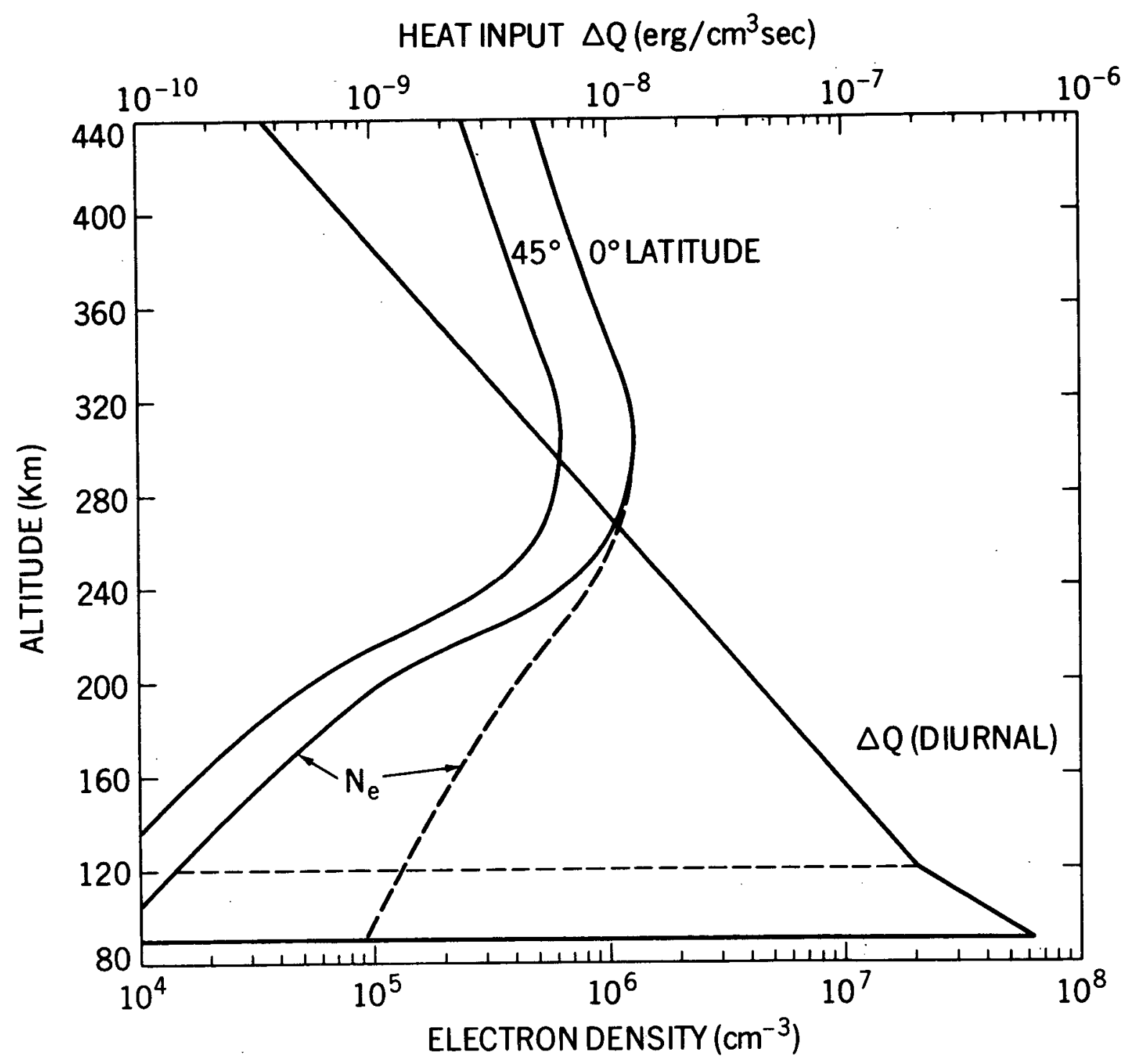

Figure 1. Electron density distributions for $0^{\circ}$ and $45^{\circ}$ latitudes. The profile in dashed line was used for the solution described with solid lines in Figure 3 . The heat input profile represents the amplitude of the diurnal component including an adopted source below $120 \mathrm{~km}$. 


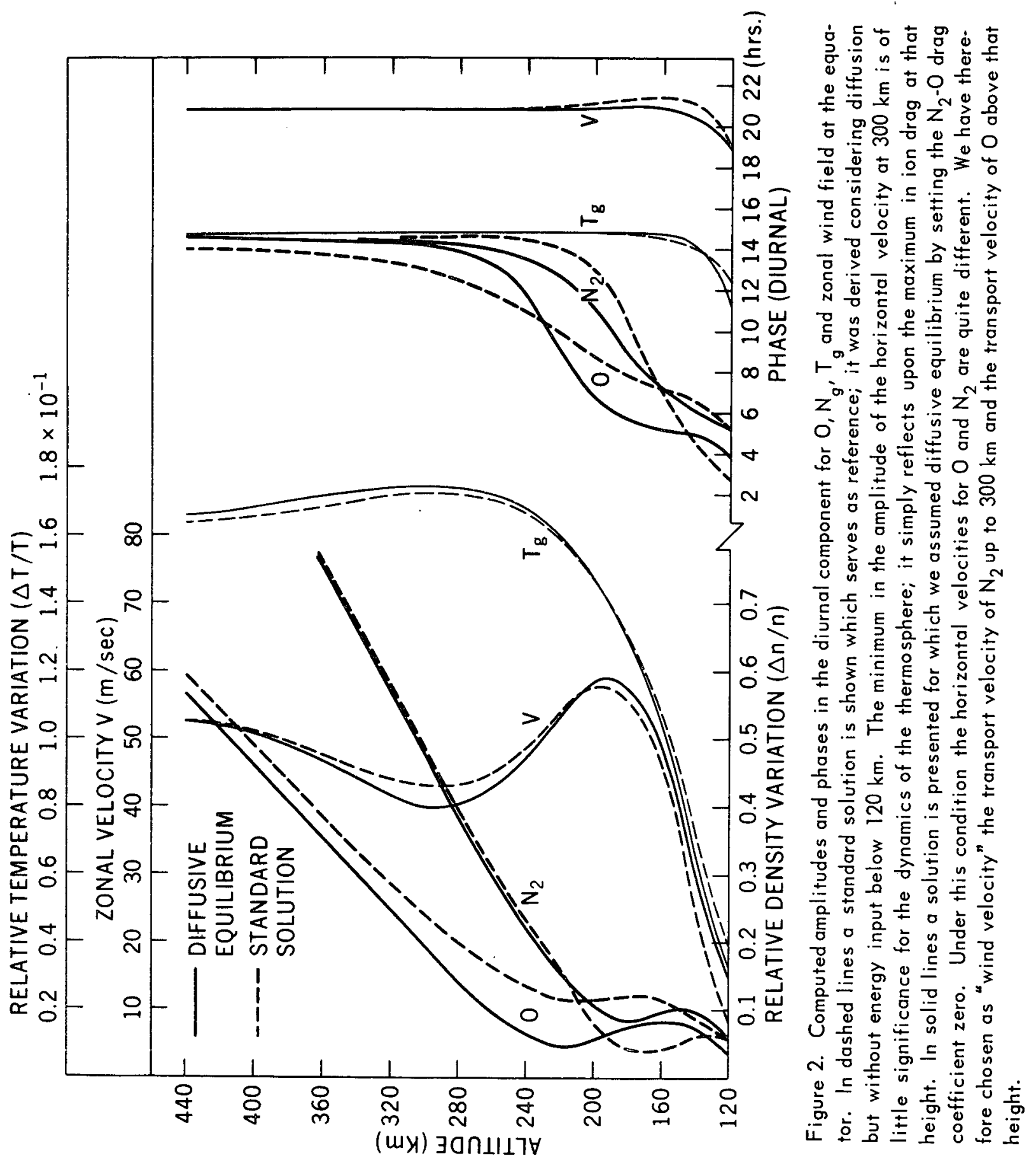




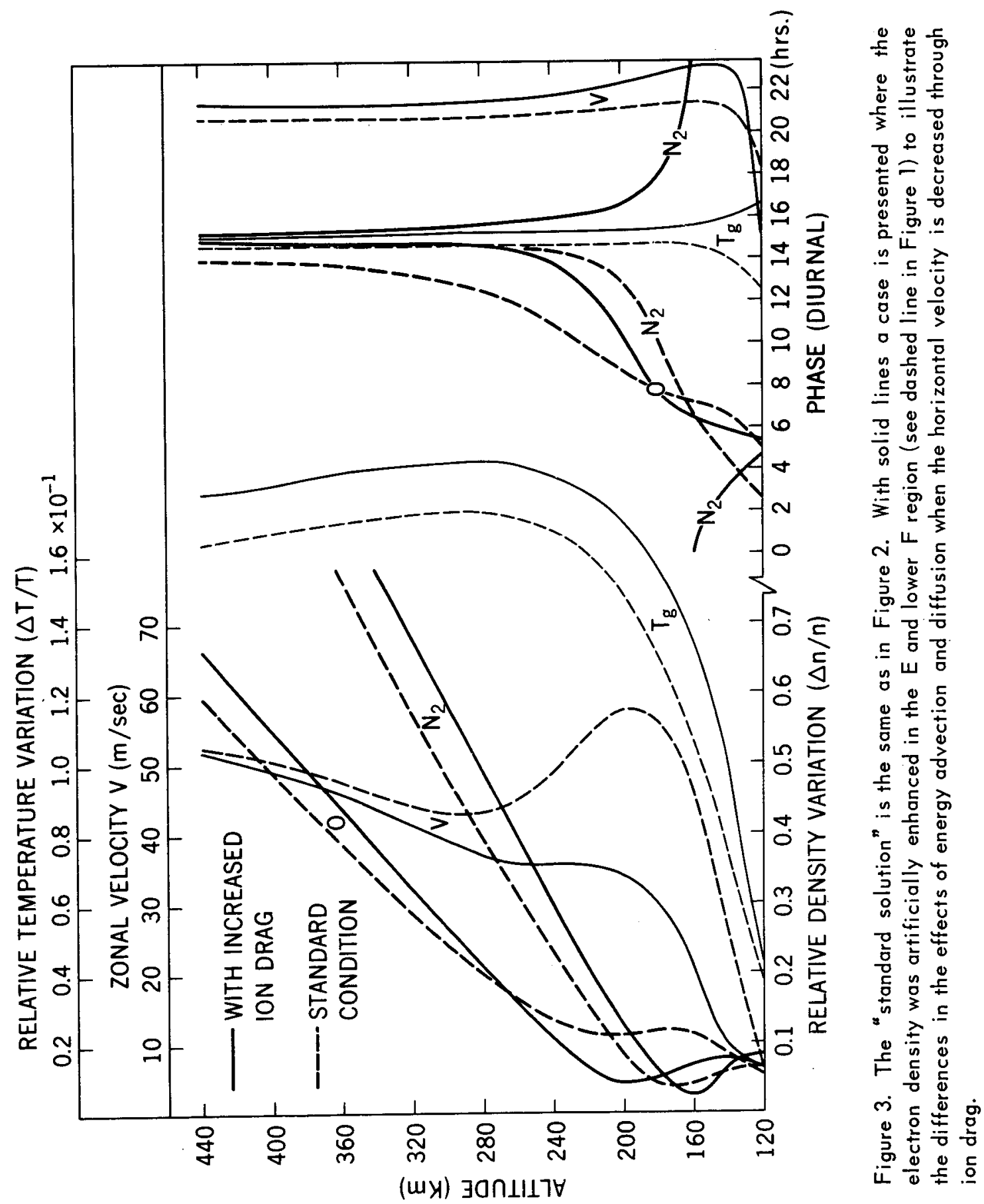




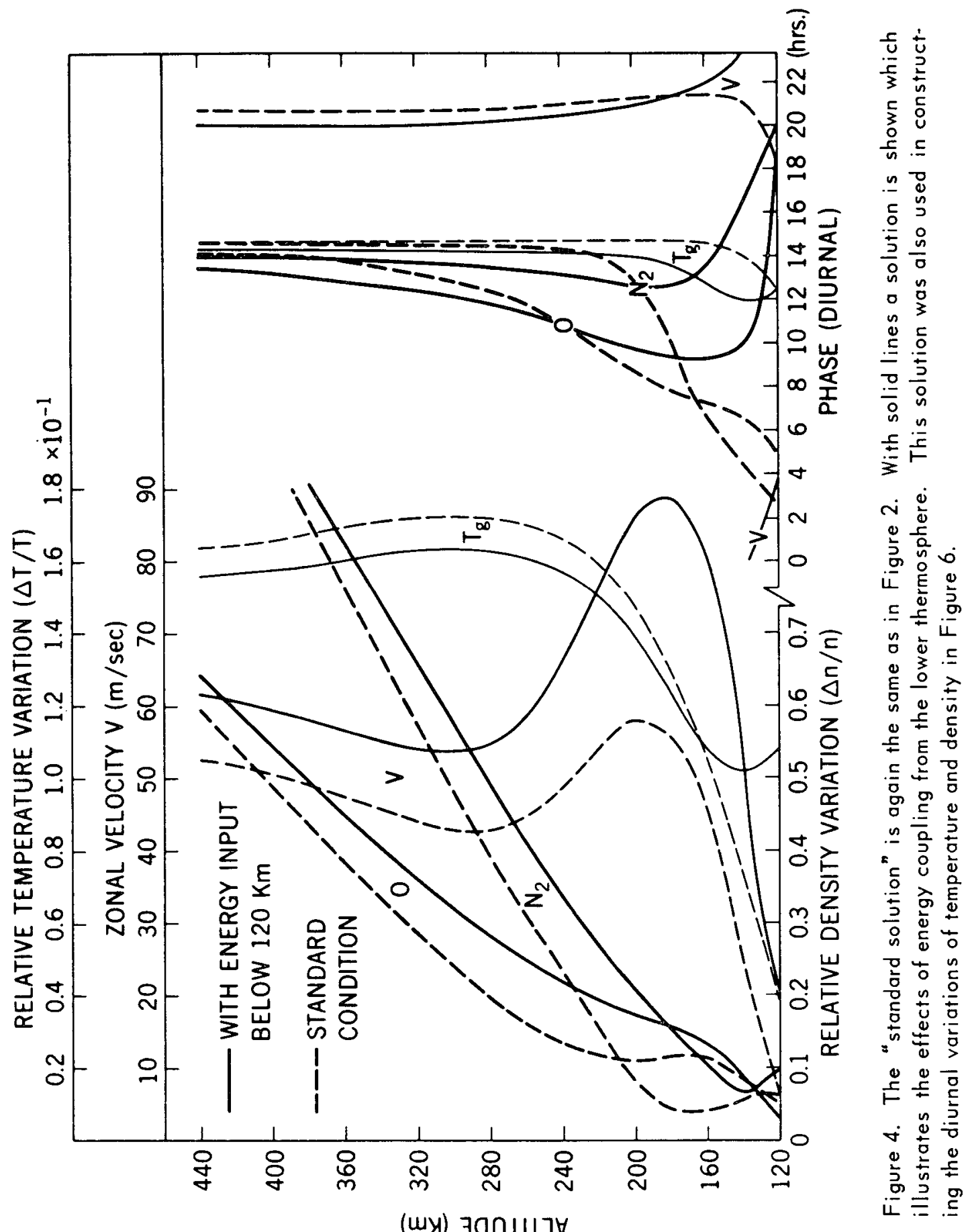

(wy) $\exists a \cap 11 \perp 7 \forall$ 


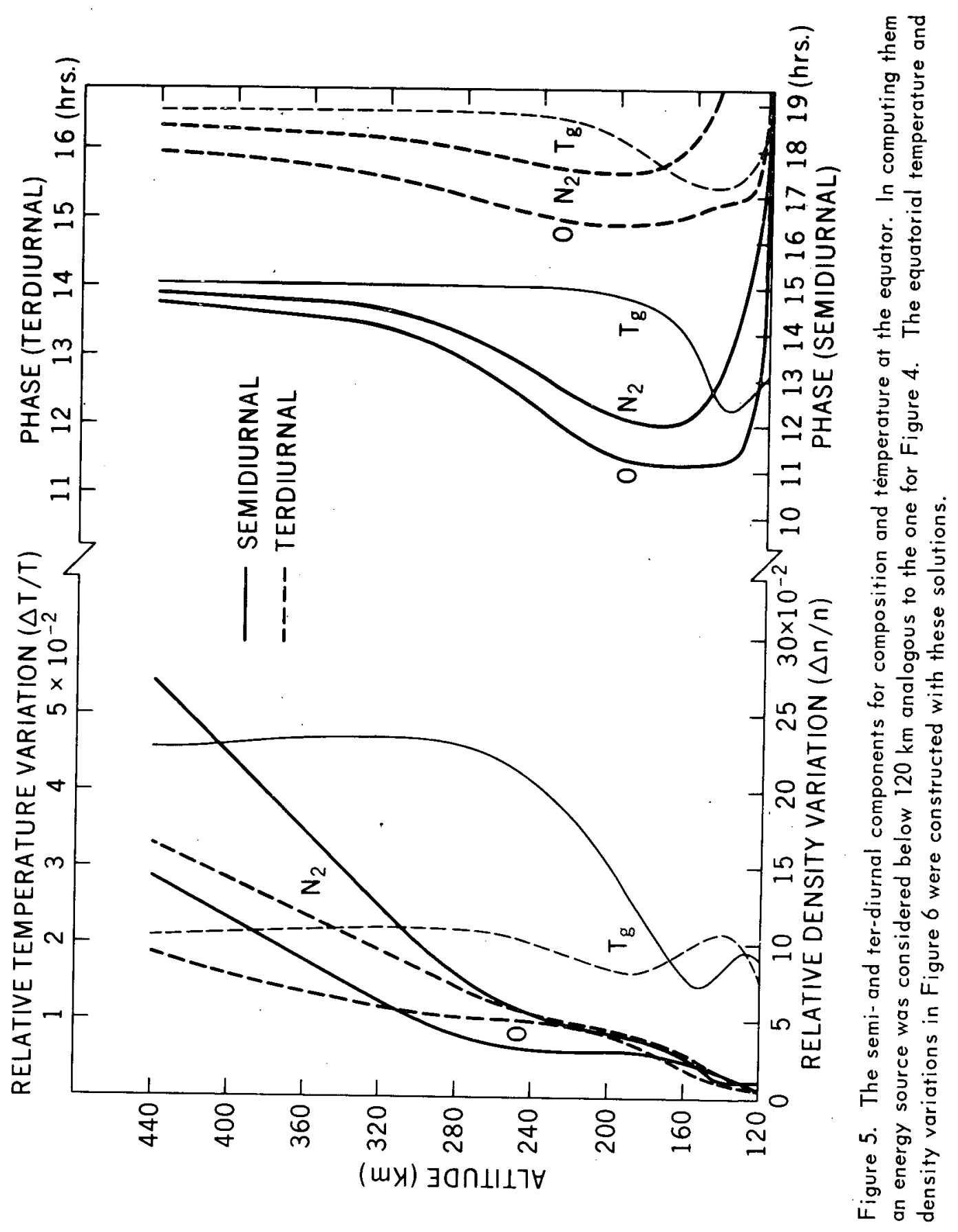

33 


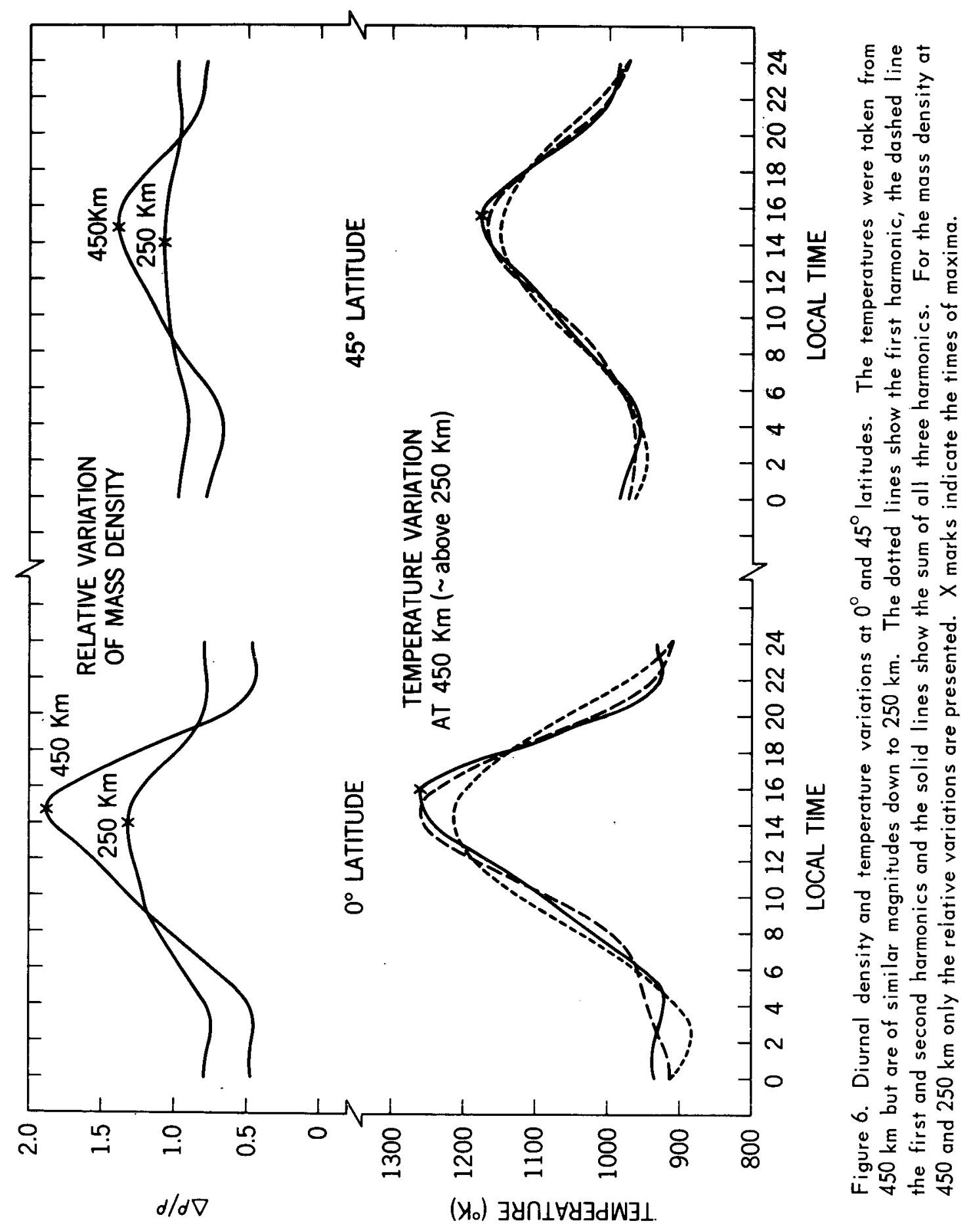

